\title{
Distributed Population Dynamics: Optimization and Control Applications
}

\author{
J. Barreiro-Gomez, G. Obando, N. Quijano
}

\begin{abstract}
Population dynamics have been widely used in the design of learning and control systems for networked engineering applications, where the information dependency among elements of the network has become a relevant issue. Classic population dynamics (e.g., replicator, logit choice, Smith, and projection) require full information to evolve to the solution (Nash equilibrium). The main reason is that classic population dynamics are deduced by assuming well-mixed populations, which limits the applications where this theory can be implemented. In this work, we extend the concept of population dynamics for nonwell-mixed populations in order to deal with distributed information structures that are characterized by non-complete graphs. Although the distributed population dynamics proposed in this paper use partial information, they preserve similar characteristics and properties of their classic counterpart. Specifically, we prove mass conservation and convergence to Nash equilibrium. To illustrate the performance of the proposed dynamics, we show some applications in the solution of optimization problems, classic games, and the design of distributed controllers.
\end{abstract}

\section{INTRODUCTION}

Population dynamics [1], [2], [3] describe the dynamical process that a population experiences when there is a strategic interaction among the agents that comprise the population. The agents involved in this dynamical process evolve to an equilibrium according to a revision protocol, which establishes the individual decision rules that agents apply to choose the best strategies (i.e., those strategies earning higher payoffs). Population dynamics properties (e.g., passivity [4]) can be exploited to design solutions for a variety of engineering problems. For instance, designing of control and learning systems [5], [6], optimization [7], [8], coordination [9], dynamic resource allocation [10], and so forth.

When using population dynamics for solving learning, control, and optimization problems, some elements of the problem are associated with "strategies" that agents in the population can adopt, and other elements are associated with "masses" of agents playing each strategy. This analogy has a direct implication in the information required to implement a solution based on a population dynamics algorithm, since the existing algorithms assume that the population is well-mixed

Manuscript received XXX. This work has been supported by COLCIENCIAS-COLFUTURO, grants No: 528 and 6172; and by Project ALTERNAR, Acuerdo 005, 07/19/13 CTeI-SGR-Nariño, Colombia.

J. Barreiro-Gomez, G. Obando and N. Quijano are with Departamento de Ingeniería Eléctrica y Electrónica, Universidad de los Andes, Cra. 1 No 18A10, Bogotá, Colombia (e-mails: \{j.barreiro135, ge-oband, nquijano\} (uniandes.edu.co)

J. Barreiro-Gomez is also with the Automatic Control Department, Universitat Politècnica de Catalunya, Institut de Robòtica i Informàtica Industrial (CSIC-UPC), Llorens i Artigas, 4-6, 08028 Barcelona, Spain. (e-mail: jbarreiro @iri.upc.edu).
[1], [3] (i.e., any pair of agents playing any pair of strategies can interact with each other). A consequence of the wellmixed population structure assumption is that the elements of the problem are allowed to interact each other without any constraint (i.e., following a full-information structure). Therefore, classic population dynamics are restricted to be implemented in problems characterized by a centralized information scheme. However, the number of problems that require distributed solutions has increased dramatically in the last few years. In this regard, some approaches have been proposed to model the interaction constraints in a population of players. For instance, the authors in [11], [12] deal with normal-form games and propose a graph-based interaction model, where each node in the graph represents an individual that repetitively plays a symmetric game with its neighbors. However, this approach is not suitable to be applied in the population game framework since, in this framework, it is preferable to avoid the individuation of players [3]. On the other hand, other approaches aim to apply learning algorithms that are capable to deal with information constraints [13], [14]. Similarly, the authors in [15] modify the well known replicator dynamics model to relax the full-information dependency. They propose a distributed replicator equation in which the evolution of each strategy is only governed by the "neighboring" strategies (according to a given topology).

This paper extends the results in [15]. Our main contribution is the design of a general method that allows us to deduce several distributed population dynamics. The core of the proposed method is the use of the mean dynamics [3] in nonwell-mixed populations. To illustrate our methodology, we present a distributed version of the fundamental population dynamics (those obtained by applying classic revision protocols), i.e., the distributed replicator dynamics, the distributed Smith dynamics, the distributed logit dynamics, and the distributed projection dynamics. It is worth noting that the deduction presented in this work can be used to generate other distributed dynamics by using alternative revision protocols. Besides, we show that a well-mixed population obeys a structure given by a complete graph, whereas a non-well-mixed population has many different possible structures that are generally given by non-complete graphs. In this sense, the proposed approach is versatile to be implemented in a large variety of problems with different information structures. Moreover, we show that the distributed population dynamics exhibit similar stability and invariance properties as their classic counterpart. Finally, the application of the deduced distributed population dynamics in optimization problems, classic games, and controllers design is highlighted. 
The remainder of this paper is organized as follows. Section II presents some preliminary concepts related to population games and graphs. Section III introduces the mean dynamics in non-well-mixed populations. Section III-B shows the deduction of distributed population dynamics from the mean dynamics and several revision protocols. In Section IV-A, the invariant set analysis corresponding to the population mass constraint is presented. Section IV-B derives sufficient conditions on the graph associated with the population structure that guarantee the convergence of the distributed population dynamics to a Nash equilibrium. Section V presents some examples that illustrate the application of the proposed dynamics in: $i$ ) distributed optimization, $i i)$ classic games, and iii) design of distributed controllers. Finally, results are discussed in Section VI, and concluding remarks are drawn in Section VII.

\section{PRELIMINARIES}

\section{A. Graphs}

We use graph-theoretical tools to describe the constraints on the interactions among agents according to the population type, i.e., well-mixed or non-well-mixed. In this regard, let $\mathcal{G}=(\mathcal{V}, \mathcal{E}, \mathcal{A})$ be a weighted graph, where $\mathcal{V}=\{1, \ldots, n\}$ is the set of nodes representing the strategies in the population; $\mathcal{E} \subset\{(i, j): i, j \in \mathcal{V}\}$ is the set of links representing possible interaction among agents playing different strategies; and $\mathcal{A}=$ $\left[a_{i j}\right]$ is an $n \times n$ weighted adjacency matrix whose elements satisfy the following property: $a_{i j}>0$ if $(i, j) \in \mathcal{E}, a_{i j}=0$ otherwise. Furthermore, the set of neighbors of a node $i \in \mathcal{V}$, which is denoted by $\mathcal{N}_{i}$, is defined as $\mathcal{N}_{i}=\{j:(i, j) \in \mathcal{E}\}$.

\section{B. Population Games}

Consider a population of mass $m>0$ comprised of a finite and large number of agents in a strategic interaction. Throughout this paper, we assume without loss of generality, that the mass of the population is equal to one, i.e., $m=1$. As was stated before, the set of available strategies for the agents is given by $\mathcal{V}=\{1, \ldots, n\}$. The scalar $x_{i} \geq 0$ represents the fraction of the population mass that corresponds to the agents choosing the strategy $i \in \mathcal{V}$. The vector $x \in \mathbb{R}_{+}^{n}$ is the state of the population with dimension $n$ whose entries are nonnegative real numbers. Similarly, $x \in \mathbb{R}_{++}^{n}$ denotes a vector of dimension $n$ with strictly positive entries. The set of possible states of the population, which corresponds to all possible distributions of agents among the strategies, is given by the following simplex:

$$
\Delta=\left\{x \in \mathbb{R}_{+}^{n}: \sum_{i \in \mathcal{V}} x_{i}=1\right\} .
$$

Agents playing the $i$ th strategy obtain a reward given by a fitness function $f_{i}(x)$, i.e., $f_{i}: \Delta \rightarrow \mathbb{R}$ is a continuous map that specifies the payoff associated with the strategy $i \in \mathcal{V}$. Notice that a population game is completely characterized by the fitness vector $F(x)=\left[f_{1}(x), \ldots, f_{n}(x)\right]^{\top}$. There are several types of games depending on $F(x)$. Below, we present the definition of two of them, which are found in a large number of applications [16].
Definition 1. Let $F: \mathbb{R}_{+}^{n} \mapsto \mathbb{R}^{n}$ be a population game with payoffs defined on the positive orthant. If there exists a continuously differentiable potential function $V: \mathbb{R}_{+}^{n} \mapsto \mathbb{R}$ that satisfies $\nabla V(x)=F(x)$, for all $x \in \mathbb{R}_{+}^{n}$, then $F$ is a full potential game.

Definition 2. The population game $F: \Delta \mapsto \mathbb{R}^{n}$ is a stable game if: $(y-x)^{\top}(F(y)-F(x)) \leq 0$, for all $x, y \in \Delta$.

The next theorem, adapted from [3], gives an equivalent characterization of a stable game in terms of the Jacobian matrix of $F(x)$, i.e., $D F(x)$; and the tangent space of the simplex $\Delta$.

Theorem 1. Let the population game $F: \Delta \mapsto \mathbb{R}^{n}$ be continuously differentiable. $F$ is a stable game if and only if $\xi^{\top} D F(x) \xi \leq 0$, for all $\xi \in T \Delta, x \in \Delta$, where $T \Delta$ is the tangent space of the simplex $\Delta$, which is defined by $T \Delta=\left\{z \in \mathbb{R}^{n}: \sum_{i \in \mathcal{V}} z_{i}=0\right\}$.

A population game combined with a revision protocol lead to the emergence of population dynamics [3]. The function $\rho: \mathbb{R}^{n} \times \Delta \rightarrow \mathbb{R}_{+}^{n \times n}$ is known as the revision protocol, and describes the timing and the result of the decisions of agents in the strategic interaction. The revision protocol takes the payoff vector $F(x)$ and a determined population state $x \in \Delta$, returning a non-negative matrix, whose element of the $i$ th row and $j$ th column $\rho_{i j}(F(x), x)$ represents the conditional switch rate from strategy $i$ to strategy $j$, where $i, j \in \mathcal{V}$. Depending on the revision protocol used by the individuals, we can find several kinds of population dynamics (see Table I), e.g., replicator dynamics, Smith dynamics, logit dynamics, projection dynamics, etc.

Table I

SOME REVISION PROTOCOLS AND THEIR CORRESPONDING POPULATION DYNAMICS [3].

\begin{tabular}{lll}
\hline Revision protocol & Population dynamics \\
\hline \hline PPIP: & $\rho_{i j}=x_{j}\left[f_{j}-f_{i}\right]_{+}$ & Replicator dynamics \\
PCP: & $\rho_{i j}=\left[f_{j}-f_{i}\right]_{+}$ & Smith dynamics \\
LCP: & $\rho_{i j}=\frac{e^{\eta^{-1} f_{j}}}{\sum_{k \in \mathcal{V}} e^{\eta^{-1} f_{k}}}, \eta>0$ & Logit dynamics \\
& & \\
MPCP: & $\rho_{i j}=\frac{\left[f_{j}-f_{i}\right]_{+}}{x_{i}}$ & Projection dynamics \\
\hline
\end{tabular}

$[\cdot]_{+}:=\max (\cdot, 0)$

PPIP: Pairwise proportional imitation protocol, PCP: Pairwise comparison protocol, LCP: Logit choice protocol, MPCP: Modified pairwise comparison.

In order to simplify the notation for fitness functions, and revision protocols, we remove their corresponding arguments from now on, i.e, $f_{i}=f_{i}(x)$, and $\rho_{i j}=\rho_{i j}(F(x), x)$.

\section{GENERAL DYNAMICS ON GRAPHS}

The dynamics describing a population behavior depend on the population structure. In this regard, current literature assumes that the population under consideration is wellmixed, i.e., if we take any portion of the entire population, this contains all the strategies with the same probability. Figure 1a illustrates this fact by showing a population composed by a large and finite number of agents involved in a game. Each element in the figure represents an agent, and the shape of the 
element ("circle", "square", or "triangle") denotes the strategy that the agent has adopted. In population games, all agents have the same probability to receive a revision opportunity. The agent receiving the revision opportunity randomly choose another agent from its neighbors and can change its own strategy by the neighbor's strategy depending on the selected revision protocol. Since the population is well-mixed, the probability that the selected opponent is playing any of the available strategies is the same.

On the other hand, there could be a non-well-mixed population as the one shown in Figure 1b. For this population, all agents have the same probability to receive an opportunity to make a revision. However, the probability that the opponent is playing a particular strategy is not equal (e.g., if the strategy played by the agent receiving the revision opportunity is "square", then there is the same probability to select an opponent playing strategy "triangle" or "square"; but the probability to select an opponent playing strategy "circle" is zero since the population structure does not allow it).

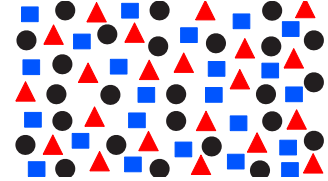

a)

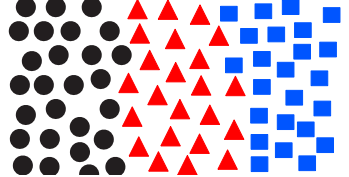

b)
Figure 1. a) Well-mixed population. b) Non-well-mixed population.

Interactions among agents playing different strategies can be represented by a graph $\mathcal{G}=\{\mathcal{V}, \mathcal{E}, \mathcal{A}\}$. The set of nodes $\mathcal{V}$ is associated with the available strategies and the set of links $\mathcal{E}$ is related to the encounter probability between strategies, i.e., there exists a link between two strategies if their encounter probability is different from zero. Hence, the elements of the corresponding adjacency matrix $\mathcal{A}=\left[a_{i j}\right]$ are as follows: $a_{i j}=1$ denotes that strategies $i$ and $j$ can encounter each other, while $a_{i j}=0$ denotes that the population structure makes impossible a matching between strategies $i$ and $j$. According to this convention, the scenarios associated with wellmixed and non-well-mixed populations can be represented by two kinds of graphs. The well-mixed population case is always represented by a complete graph, whereas a non-well-mixed population is represented by a graph with a specific topology depending on the particular population structure (see Figure 2). In this paper, we assume that the encounter probability between strategies $i$ and $j$ is the same as the one of strategies $j$ and $i$, i.e., the graph $\mathcal{G}$ is undirected.

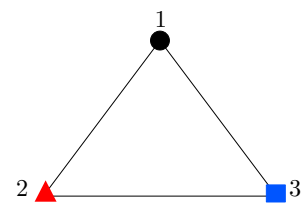

a)

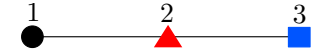

b)
Figure 2. Graph representation of: a) The well-mixed population in Figure 1a. b) The non-well-mixed population in Figure 1b. Nodes 1,2, and 3 correspond, respectively, to strategies "circle", "triangle", and "square" of the proposed example.

\section{A. Mean Dynamics}

Taking into account the previously discussed considerations, this section formally describes the evolutionary process of a non-well-mixed population involved in a strategic game. Suppose that the population is composed by $M$ agents, and each of them receives a revision opportunity that is given by an exponential distribution with rate $R$. Hence, during a time $d t$, the revision opportunity received by each agent is given by $R d t$. Since we assume that the mass of the population is equal to one, the scalar $x_{i}$ is equal to the portion of agents playing the $i$ th strategy, and $M x_{i}$ is the total amount of agents playing strategy $i \in \mathcal{V}$. Consequently, the expected number of revision opportunities received by agents playing the $i$ th strategy is approximately $M x_{i} R d t$ during $d t$ (notice that $x_{i}$ may vary during $d t$; however, this variation is negligible if $d t$ is small). Agents playing $i \in \mathcal{V}$ switch to strategy $j \in \mathcal{V}$ with a probability that depends on the revision protocol, the probability distribution of receiving a revision opportunity, and the encounter probability between strategies $i$ and $j$ (given by the population structure, which is represented by the graph $\mathcal{G})$, i.e., $a_{i j} \rho_{i j} / R$. Finally, the expected number of agents switching from strategy $i \in \mathcal{V}$ to strategy $j \in \mathcal{V}$ during time $d t$ is $M x_{i} a_{i j} \rho_{i j} d t$.

Now, if we consider all possible strategies in the population, the expected number of agents switching to strategy $i \in \mathcal{V}$ is given by $M \sum_{j \in \mathcal{V}} x_{j} a_{j i} \rho_{j i} d t$, and the expected number of agents playing strategy $i \in \mathcal{V}$ changing to other strategies is given by $M x_{i} \sum_{j \in \mathcal{V}} a_{i j} \rho_{i j} d t$. Therefore, the variation of the proportion of agents playing the $i$ th strategy is deduced by a mass balance as follows $\dot{x}_{i}=\sum_{j \in \mathcal{V}} x_{j} a_{j i} \rho_{j i}-$ $x_{i} \sum_{j \in \mathcal{V}} a_{i j} \rho_{i j}$. This equation corresponds to the Distributed Mean Dynamics, or mean dynamics for non-well-mixed populations. Since $\mathcal{G}$ is undirected, notice that the distributed mean dynamics can be rewritten as follows,

$$
\dot{x}_{i}=\sum_{j \in \mathcal{N}_{i}} x_{j} \rho_{j i}-x_{i} \sum_{j \in \mathcal{N}_{i}} \rho_{i j} .
$$

For complete graphs, i.e., for well-mixed-populations, we have that $\mathcal{N}_{i}=\mathcal{V}$, obtaining the classic Mean Dynamics [3].

\section{B. Distributed Population Dynamics}

Distributed mean dynamics allow the inference of population dynamics involving non-well-mixed populations comprised of agents that are programmed with a specific revision protocol. This section shows the deduction of different distributed population dynamics using (2). The deduced dynamics are named after the classic population dynamics that are generated with the corresponding revision protocol.

1) Distributed Replicator Dynamics (DRD): The distributed replicator dynamics are obtained from the distributed mean dynamics using the pairwise proportional imitation protocol (Table I), as follows,

$$
\begin{aligned}
\dot{x}_{i} & =\sum_{j \in \mathcal{N}_{i}} x_{j} x_{i}\left[f_{i}-f_{j}\right]_{+}-x_{i} \sum_{j \in \mathcal{N}_{i}} x_{j}\left[f_{j}-f_{i}\right]_{+} \\
& =\sum_{j \in \mathcal{N}_{i}} x_{j} x_{i}\left(f_{i}-f_{j}\right) .
\end{aligned}
$$


Finally, the distributed replicator dynamics are given by,

$$
\dot{x}_{i}=x_{i}\left(f_{i} \sum_{j \in \mathcal{N}_{i}} x_{j}-\sum_{j \in \mathcal{N}_{i}} x_{j} f_{j}\right) .
$$

2) Distributed Smith Dynamics (DSD): In this case, we use the pairwise comparison protocol (Table I). Substituting this revision protocol in (2), we get

$$
\dot{x}_{i}=\sum_{j \in \mathcal{N}_{i}} x_{j}\left[f_{i}-f_{j}\right]_{+}-x_{i} \sum_{j \in \mathcal{N}_{i}}\left[f_{j}-f_{i}\right]_{+} \cdot
$$

Notice that (4) can be written as,

$$
\dot{x}_{i}=\sum_{j \in \mathcal{N}_{i}} \frac{1}{2}\left(\left(1-\phi_{i j}\right) x_{i}+\left(1+\phi_{i j}\right) x_{j}\right)\left[f_{i}-f_{j}\right],
$$

where $\phi_{i j}=\operatorname{sgn}\left(f_{i}-f_{j}\right)$.

3) Distributed Logit Dynamics (DLD): The deduction of the distributed logit dynamics is based on the logit choice protocol (Table I). However, notice that this protocol requires full information since the sum at the denominator is taken over all the strategies. In order to satisfy the information constraint given by the graph $\mathcal{G}$, we modify the protocol as follows,

$$
\rho_{i j}(F, x)=e^{\eta^{-1} f_{j}}, \eta>0 .
$$

Distributed logit dynamics are obtained by replacing the above protocol in the distributed mean dynamics, i.e.,

$$
\dot{x}_{i}=\sum_{j \in \mathcal{N}_{i}} x_{j} e^{\eta^{-1} f_{i}}-x_{i} \sum_{j \in \mathcal{N}_{i}} e^{\eta^{-1} f_{j}} .
$$

4) Distributed Projection Dynamics (DPD): The projection dynamics use the modified pairwise comparison protocol, i.e.,

$$
\begin{aligned}
\dot{x}_{i} & =\sum_{j \in \mathcal{N}_{i}} x_{j} \frac{\left[f_{i}-f_{j}\right]_{+}}{x_{j}}-x_{i} \sum_{j \in \mathcal{N}_{i}} \frac{\left[f_{j}-f_{i}\right]_{+}}{x_{i}}, \\
& =\sum_{j \in \mathcal{N}_{i}}\left(f_{i}-f_{j}\right) .
\end{aligned}
$$

Thus, the distributed projection dynamics are given by

$$
\dot{x}_{i}=\left|\mathcal{N}_{i}\right| f_{i}-\sum_{j \in \mathcal{N}_{i}} f_{j},
$$

where $\left|\mathcal{N}_{i}\right|$ denotes the cardinality of the set $\mathcal{N}_{i}$, i.e., the number of neighbors of the $i$ th node.

\section{INVARIANT SET AND StABILITy ANALYSIS}

\section{A. Invariant Set Analysis}

As was mentioned in Section II, the population mass does not vary over time. Hence, all possible states generated during the evolution of the population should belong to the simplex $\Delta$ given in (1). This section shows that the simplex $\Delta$ is an invariant set under the distributed population dynamics deduced in the previous section.

Theorem 2. The simplex $\Delta$ is an invariant set under: the distributed replicator dynamics (3), the distributed Smith dynamics (4), and the distributed logit dynamics (5).
Proof. According to Equation (1), $\Delta$ has to conditions: i) $\sum_{i \in \mathcal{V}} x_{i}=1$ (mass conservation); ii) $x_{i} \geq 0$, for all $i \in \mathcal{V}$ (non-negativeness).

First, we prove that DRD, DSD, and DLD satisfy condition i). Notice that this is equivalent to show that $\sum_{i \in \mathcal{V}} \dot{x}_{i}=0$ under the distributed mean dynamics (2). These dynamics can be written by using the adjacency matrix of the graph $\mathcal{G}$ as follows

$$
\dot{x}_{i}=\sum_{j \in \mathcal{V}} a_{i j} \rho_{j i} x_{j}-\sum_{j \in \mathcal{V}} a_{i j} \rho_{i j} x_{i}
$$

Hence,

$$
\sum_{i \in \mathcal{V}} \dot{x}_{i}=\sum_{i \in \mathcal{V}} \sum_{j \in \mathcal{V}} a_{i j} \rho_{j i} x_{j}-\sum_{i \in \mathcal{V}} \sum_{j \in \mathcal{V}} a_{i j} \rho_{i j} x_{i} .
$$

Since $\mathcal{G}$ is undirected (i.e., $a_{i j}=a_{j i}$ ), we have

$$
\begin{aligned}
\sum_{i \in \mathcal{V}} \dot{x}_{i} & =\sum_{i \in \mathcal{V}} \sum_{j \in \mathcal{V}} a_{j i} \rho_{j i} x_{j}-\sum_{j \in \mathcal{V}} \sum_{i \in \mathcal{V}} a_{j i} \rho_{j i} x_{j} \\
& =0 .
\end{aligned}
$$

Second, we prove that each dynamic satisfies condition $i$ ):

- DRD: Non-negativeness of each $x_{i}$ is satisfied given the fact that $\dot{x}_{i}=0$ if $x_{i}=0$ under distributed replicator dynamics. Thus, if $x_{i}(0) \geq 0$, then $x_{i}(t) \geq 0$ for all $t \geq 0$.

- DSD: According to (4), notice that when $x_{i}=0$ for any $i \in \mathcal{V}$, then $\dot{x}_{i} \geq 0$. Hence, the non-negativeness of $x_{i}$ is satisfied under distributed Smith dynamics.

- DLD: Notice that $\dot{x}_{i} \geq 0$ when $x_{i}=0$ under distributed logit dynamics (5). Therefore, if $x(0) \in \Delta$, then $x_{i}(t) \geq$ 0 for all $t \geq 0$.

Proposition 1. The set $\Delta^{\prime}=\left\{x \in \mathbb{R}^{n}: \sum_{i \in \mathcal{V}} x_{i}=1\right\}$ is invariant under the distributed projection dynamics (6).

Proof. The distributed projection dynamics can be written by using the adjacency matrix of the graph $\mathcal{G}$ as follows

$$
\dot{x}_{i}=\sum_{j \in \mathcal{V}} a_{i j} f_{i}-\sum_{j \in \mathcal{V}} a_{i j} f_{j} .
$$

Therefore,

$$
\sum_{i \in \mathcal{V}} \dot{x}_{i}=\sum_{i \in \mathcal{V}} \sum_{j \in \mathcal{V}} a_{i j} f_{i}-\sum_{i \in \mathcal{V}} \sum_{j \in \mathcal{V}} a_{i j} f_{j} .
$$

Since $a_{i j}=a_{j i}$ because $\mathcal{G}$ is undirected, we obtain

$$
\begin{aligned}
\sum_{i \in \mathcal{V}} \dot{x}_{i} & =\sum_{j \in \mathcal{V}} \sum_{i \in \mathcal{V}} a_{j i} f_{i}-\sum_{i \in \mathcal{V}} \sum_{j \in \mathcal{V}} a_{i j} f_{j} \\
& =0 .
\end{aligned}
$$

Remark 1. It should be noticed that the distributed projection dynamics satisfy one of the conditions of the original simplex $\Delta$, i.e., $\sum_{i \in \mathcal{V}} x_{i}=1$ (mass conservation). However, the nonnegativeness of $x_{i}$ is not guaranteed. This fact also occurs in the classic projection dynamics.

Remark 2. Notice that Theorem 2 and Proposition 1 do not impose any conditions on the interaction graph $\mathcal{G}$. Thus, the studied distributed population dynamics exhibit simplex invariance under any population structure. 


\section{B. Stability Analysis}

Classic population dynamics usually converge to Nash equilibria since Nash equilibria correspond to the expected outcome of games played by rational individuals (i.e., individuals that are trying to maximize their profit). Given a population game $F$, the set of Nash equilibria is defined as follows [3],

$$
N E(F)=\left\{x^{*} \in \Delta: x_{i}^{*}>0 \Rightarrow f_{i}\left(x^{*}\right) \geq f_{j}\left(x^{*}\right), \forall i, j \in \mathcal{V}\right\} .
$$

Thus, in a Nash equilibrium, all players perceives the same profit.

This section provides sufficient conditions guaranteeing that a Nash equilibrium $x^{*}$ of the population game $F$ is asymptotically stable under the distributed population dynamics derived in Section III-B. These conditions, which are related to the connectivity of the interaction graph and the characteristics of the Nash equilibrium, are summarized in the following assumptions.

Assumption 1. The graph $\mathcal{G}$ that describes the population structure is connected.

Assumption 2. The Nash equilibrium $x^{*}$ belongs to the interior of the simplex $\Delta$, i.e., $x^{*} \in$ int $\Delta$, where int $\Delta=\left\{x \in \mathbb{R}_{++}^{n}: \sum_{i \in \mathcal{V}} x_{i}=1\right\}$.

Below, we provide our results on convergence of the distributed population dynamics to a Nash equilibrium.

Theorem 3. Let $F$ be a full potential game with strictly concave potential function $V(x)$, and let $x^{*} \in N E(F)$. If Assumptions 1 and 2 hold, then $x^{*}$ is asymptotically stable under the distributed replicator dynamics (3) and the distributed Smith dynamics (4).

Proof. Since $x^{*} \in N E(F)$ and $x^{*} \in \operatorname{int} \Delta$, we conclude that $f_{i}\left(x^{*}\right)=f_{j}\left(x^{*}\right)$, for all $i, j \in \mathcal{V}$. Moreover, notice that $x^{*}=\arg \max _{x \in \Delta} V(x)$ (applying the Karush-Kuhn-Tucker conditions). Additionally, since $V(x)$ is strictly concave, we can take $E_{V}(x)=V\left(x^{*}\right)-V(x)$ as a Lyapunov function candidate. The derivative of $E_{V}(x)$ along the trajectories of DRD (3) and DSD (4) is given by

$$
\begin{aligned}
\dot{E}_{V}(x) & =-(\nabla V(x))^{\top} \dot{x} \\
& =-F^{\top} \dot{x} \\
& =-F^{\top} L^{(x)} F,
\end{aligned}
$$

where $L^{(x)}=\left[l_{i j}^{(x)}\right]$ is a matrix whose entries $l_{i j}^{(x)}$ are for DRD as follows:

$$
l_{i j}^{(x)}=\left\{\begin{array}{l}
-a_{i j} x_{i} x_{j}, \text { if } i \neq j \\
\sum_{k \in \mathcal{V}, k \neq i} a_{i k} x_{i} x_{k}, \text { if } i=j,
\end{array}\right.
$$

and for DSD:

$$
l_{i j}^{(x)}=\left\{\begin{array}{l}
-\frac{a_{i j}}{2}\left(\left(1-\phi_{i j}\right) x_{i}+\left(1+\phi_{i j}\right) x_{j}\right), \text { if } i \neq j \\
\sum_{k \in \mathcal{V}, k \neq i} \frac{a_{i k}}{2}\left(\left(1-\phi_{i k}\right) x_{i}+\left(1+\phi_{i k}\right) x_{k}\right), \text { if } i=j .
\end{array}\right.
$$

Notice that $L^{(x)}$ is the Laplacian of the undirected graph given by the tuple $\mathcal{G}^{(x)}=\left(\mathcal{V}, \mathcal{E}, \mathcal{A}^{(x)}\right)$, where $\mathcal{A}^{(x)}=$ $\left[a_{i j}^{(x)}\right]$ is the adjacency matrix whose entries are defined as follows: $a_{i j}^{(x)}=a_{i j} x_{i} x_{j}$, for DRD; and $a_{i j}^{(x)}=$ $\frac{a_{i j}}{2}\left(\left(1-\phi_{i j}\right) x_{i}+\left(1+\phi_{i j}\right) x_{j}\right)$, for DSD. These entries are nonnegative since $x \in \Delta$. Thus, $L^{(x)} \geq 0$ and $\dot{E}_{V}(x) \leq 0$. Therefore, $x^{*}$ is stable under DRD and DSD.

Considering that $x^{*} \in \operatorname{int} \Delta$ is stable, a set $\mathcal{B}$ around $x^{*}$ can be defined such that if $x(0) \in \mathcal{B}$, then $x(t) \in$ int $\Delta$, for all $t \geq 0$ (it is possible to show that $\mathcal{B}=\operatorname{int} \Delta$ for DRD). Thus, if $x(0) \in \mathcal{B}$, the null space of $L^{(x)}$ is equal to $\operatorname{span}\{\mathbb{1}\}$ (we use $\mathbb{1}$ to denote a vector of dimension $n$ whose entries are all 1) since $\mathcal{G}^{(x)}$ is connected (we conclude that $\mathcal{G}^{(x)}$ is connected since: i) $\mathcal{G}^{(x)}$ and $\mathcal{G}$ have the same topology in $\mathcal{B}$, i.e., if $x \in \mathcal{B}, a_{i j}^{(x)}=0$ only if $a_{i j}=0$; ii) $\mathcal{G}$ is connected by assumption). In this case, $\dot{E}_{V}(x)=0$ if and only if $f_{i}=f_{j}$, for all $i, j \in V$, i.e., $\dot{E}_{V}(x)=0$ only in $x^{*}$. Therefore, $x^{*}$ is asymptotically stable.

Remark 3. Theorem 3 requires that, in steady state, all strategies are played by the individuals involved in the game. Indeed, when any proportion of individuals is extinct at equilibrium (i.e., $x_{i}^{*}=0$ for some $i \in \mathcal{V}$ ), then convergence of the distributed replicator equation and the distributed Smith dynamics to the Nash equilibrium is not guaranteed. However, the same arguments used in [15] can be employed to relax the convergence conditions. In fact, if subtracting from the original graph any set of nodes associated to extinct strategies does not produce disconnected subgraphs, then convergence to Nash equilibria is provable even if Nash equilibria do not belong to the interior of $\Delta$. This relaxed assumption often holds in well-connected graphs.

Remark 4. Notice that Theorem 3 is only applicable to full potential games. However, this class of games arises in a large number of applications including resource allocation problems and congestion games [3].

Theorem 4. Let $F$ be a continuously differentiable stable game, let $x^{*} \in N E(F)$, and let $\dot{x}$ be the distributed projection dynamics (6). If Assumptions 1 and 2 hold, then $x^{*}$ is asymptotically stable.

Proof. Consider the pairwise comparison protocol $\rho_{i j}=\left[f_{j}-f_{i}\right]_{+}$, and define $\rho_{i j}=\varphi\left(f_{j}-f_{i}\right)$, where $\varphi(\cdot)=[\cdot]_{+}$. Then, consider the Lyapunov function candidate:

$$
V(x)=\sum_{i \in \mathcal{V}} \sum_{j \in \mathcal{V}} a_{i j} \int_{0}^{f j-f i} \varphi(s) d s .
$$

Since $\varphi: \mathbb{R} \mapsto \mathbb{R}_{+}$is increasing on $[0,+\infty)$ and $\mathcal{G}$ is connected, the function $V(x)>0$, for all $x \neq x^{*}$. Additionally, $V\left(x^{*}\right)=0$ since $f_{j}\left(x^{*}\right)=f_{i}\left(x^{*}\right)$, for all $i, j \in \mathcal{V}$. Moreover, notice that

$$
\begin{aligned}
& \frac{\partial V(x)}{\partial x_{l}}=\sum_{i \in \mathcal{V}} \sum_{j \in \mathcal{V}} a_{i j}\left(\frac{\partial f_{j}}{\partial x_{l}}-\frac{\partial f_{i}}{\partial x_{l}}\right) \varphi\left(f_{j}-f_{i}\right) \\
= & \sum_{i \in \mathcal{V}} \sum_{j \in \mathcal{V}} a_{i j} \varphi\left(f_{j}-f_{i}\right) \frac{\partial f_{j}}{\partial x_{l}}-\sum_{j \in \mathcal{V}} \sum_{i \in \mathcal{V}} a_{j i} \varphi\left(f_{i}-f_{j}\right) \frac{\partial f_{j}}{\partial x_{l}} .
\end{aligned}
$$


Taking into account that $a_{i j}=a_{j i}$, we obtain

$$
\begin{aligned}
\frac{\partial V(x)}{\partial x_{l}} & =\sum_{i \in \mathcal{V}} \sum_{j \in \mathcal{V}} a_{j i}\left(f_{j}-f_{i}\right) \frac{\partial f_{j}}{\partial x_{l}} \\
& =\sum_{j \in \mathcal{V}} \frac{\partial f_{j}}{\partial x_{l}} \sum_{i \in \mathcal{N}_{j}}\left(f_{j}-f_{i}\right) .
\end{aligned}
$$

According to (6), notice that $\sum_{i \in \mathcal{N}_{j}}\left(f_{j}-f_{i}\right)=\dot{x}_{j}$, where $\dot{x}_{j}$ is the $j$ th element of the distributed projection dynamics $\dot{x}$. Hence,

$$
\frac{\partial V(x)}{\partial x_{l}}=\sum_{j \in \mathcal{V}} \dot{x}_{j} \frac{\partial f_{j}}{\partial x_{l}} .
$$

Therefore, the time derivative of the Lyapunov function is

$$
\begin{aligned}
\dot{V}(x) & =(\nabla V(x))^{\top} \dot{x} \\
& =\dot{x}^{\top} D F(x) \dot{x},
\end{aligned}
$$

where $\dot{x}^{\top} D F(x) \dot{x} \leq 0$ since $F$ is stable.

Remark 5. As was stated in the proof of Theorem 3, the fact that a Nash equilibrium $x^{*}$ belongs to int $\Delta$ implies that all the fitness functions reach the same value, i.e., $f_{i}\left(x^{*}\right)=f_{j}\left(x^{*}\right)$, for all $i, j \in \mathcal{V}$. Therefore, the results given in Theorems 3 and 4 are related to the contributions reported in the literature on consensus in multi-agent networks (e.g., see [17], [18], [19]). An essential difference is that Theorems 3 and 4 show a direct relationship between game-theoretic properties and Lyapunov stability of a population game under distributed dynamics. $\diamond$

The connectivity condition of the graph $\mathcal{G}$ in Theorems 3 and 4 is sufficient for Nash equilibrium stability. Regarding this fact, it is interesting to study if this condition is also necessary. The following proposition gives us insights on this issue.

Proposition 2. Assume that the population game $F$ has a unique Nash equilibrium, which is in the interior of the simplex $\Delta$, i.e., $x^{*} \in$ int $\Delta$. Let $\dot{x}$ be the distributed mean dynamics (2). If $x(t)$ asymptotically converges to $x^{*}$, for all $x(0) \in$ int $\Delta$, then the graph $\mathcal{G}$ is connected.

Proof. We prove the contrapositive. Assume that $\mathcal{G}$ is nonconnected. We can express $\mathcal{G}$ as the union of $r \geq 2$ connected components (maximal connected sub-graphs) denoted by $\mathcal{G}^{p}=$ $\left(\mathcal{V}^{p}, \mathcal{E}^{p}\right)$, where $p=1, \ldots, r$, i.e., $\mathcal{G}=\bigcup_{p=1}^{r} \mathcal{G}^{p}$. We use the arguments in the proof of Theorem 2 to conclude that, under the distributed mean dynamics, $\sum_{i \in \mathcal{V}^{p}} x_{i}(t)=\sum_{i \in \mathcal{V}^{p}} x_{i}(0)$, for all $p=1, \ldots, r$, and for all $t \geq 0$. Take two connected components $\mathcal{G}^{1}$ and $\mathcal{G}^{2}$ of the graph $\mathcal{G}$. Furthermore, take the following initial condition:

$$
x_{i}(0)=\left\{\begin{array}{lcr}
x_{i}^{*}+\frac{\varepsilon}{\left|\mathcal{V}^{1}\right|} & \text { if } & i \in \mathcal{V}^{1} \\
x_{i}^{*}-\frac{\varepsilon}{\left|\mathcal{V}^{2}\right|} & \text { if } & i \in \mathcal{V}^{2} \\
x_{i}^{*} & \text { otherwise } &
\end{array}\right.
$$

where $i \in \mathcal{V}$, and $\varepsilon>0$. Notice that, for small values of $\varepsilon$, $x(0) \in$ int $\Delta$ since $x^{*} \in$ int $\Delta$. Under this initial condition, it is not possible that $x(t)$ converges to the unique Nash equilibrium $x^{*}$ since $\sum_{i \in \mathcal{V}^{1}} x_{i}(t)=\sum_{i \in \mathcal{V}^{1}} x_{i}(0)>\sum_{i \in \mathcal{V}^{1}} x_{i}^{*}$, for all $t \geq 0$.
Therefore, if we need to guarantee convergence to the Nash equilibrium from any initial condition inside the simplex $\Delta$, we require that the graph $\mathcal{G}$ is connected. However, this condition might not be necessary if the initial conditions $x(0)$ are constrained. For instance, suppose that the graph $\mathcal{G}$ in the population game is non-connected. Moreover, $\mathcal{G}$ is composed of $r$ connected components (maximal connected sub-graphs) denoted by $\mathcal{G}^{p}=\left(\mathcal{V}^{p}, \mathcal{E}^{p}\right)$, where $p=1, \ldots, r$, i.e., $\mathcal{G}=\bigcup_{p=1}^{r} \mathcal{G}^{p}$. Then, it can be shown (following the same reasoning as in proof of Proposition 2) that the equilibrium point $x^{*} \in$ int $\Delta$ is asymptotically stable if $\sum_{i \in \mathcal{V}^{p}} x_{i}^{*}=$ $\sum_{i \in \mathcal{V}^{p}} x_{i}(0)$ for all $p=1, \ldots, r$. Consequently, in this case, the connectivity condition of $\mathcal{G}$ is not necessary.

\section{ILLUSTRATIVE EXAMPLES}

Recently, game-theory-based techniques have received widespread attention from the engineering community, especially for addressing optimization and control problems. This fact has been mainly motivated by three reasons: i) Many engineering problems can be formulated as strategic games. Indeed, the analogy between problems' components (such as objectives, decision makers, control actions, etc.) and games' components (payoff functions, players, and strategies) is natural in several cases (e.g., see [20], [21]). In this regard, thinking of an engineering problem as a game is often useful to devise solution methods. ii) The rational outputs of games, i.e., Nash equilibria, provide proper solutions of engineering problems. In fact, there has been shown that, in some applications, there exists a complete equivalence between the optimal solution of the addressed problem and the Nash equilibrium of the underlying game [3] (a specific case for which this property holds is in potential games). iii) Solution of games can be obtained by employing local information [22], [23]. Therefore, if a game framework is applied to address an engineering problem, distributed methodologies emerge.

Among the reasons described above, the last one is of special interest in current applications. This fact is because the rapid growth of systems in complexity and scale makes centralized approaches unsuitable. Hence, distributed techniques are becoming more prevalent nowadays. This section is devoted to propose distributed solutions from the perspective of population games. It is worth noting that classic population games (which requires centralized information) have been applied to solve a large number of engineering problems. For instance, access control in communication networks [9], combinatorial optimization [24], bandwidth allocation [25], hierarchical frequency control in microgrids [26], dispatch of electric generators [27], building temperature control [10], constrained extremum seeking [28], control of drinking water networks [29], and so forth. The same applications can be addressed with the approach developed in this paper, i.e., by using distributed population dynamics. This approach has some benefits related to the information privacy, resilience to central failures, and parallelization of the computations. In order to show the versatility of the distributed population dynamics, three application examples are presented corresponding to the following key areas: distributed optimization, classic games, and distributed control design. 


\section{A. Solving a Distributed Optimization Problem}

First, we propose the following distributed optimization problem,

$$
\begin{array}{ll}
\max & V(x):=-x^{\top} x+b^{\top} x \\
\text { s.t. } & \sum_{i=1}^{50} x_{i}=1, \text { and } x_{i}>0 \text { for all } i=1, \ldots, 50,
\end{array}
$$

where $x \in \mathbb{R}^{50}$ is the vector of decision variables, and $b \in \mathbb{R}^{50}$ is a vector of constants, whose entries are given by $b_{i}=\frac{2 i}{1275}$, i.e., $b=\frac{1}{1275}\left[\begin{array}{llll}2 & 4 & \ldots & 100\end{array}\right]^{\top}$. Each decision variable is managed by a node in a network. Furthermore, we impose an information constraint given by the graph shown in Figure 3. This graph is obtained by following the ErdösRényi model (which is the simplest model of several kind of social and biological networks [30]) with edge generation probability equal to 0.01 . Besides, we add a path connecting all nodes to guarantee that the generated graph is connected. The information constraint implies that the $i$ th node only has information about the state of its neighbors. In order to

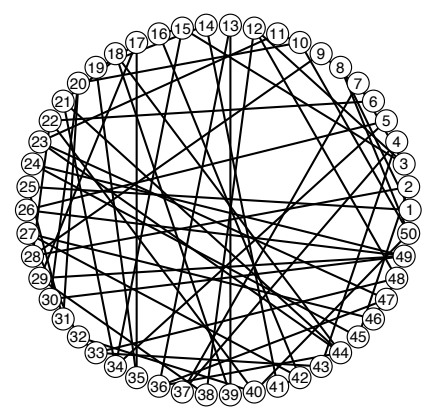

Figure 3. Non-complete graph for the distributed optimization example.

solve the problem in (8), we define a full potential game $F=\left[\frac{\partial V}{\partial x_{1}}, \ldots, \frac{\partial V}{\partial x_{50}}\right]^{\top}$ (i.e., the fitness functions correspond to the marginal utilities) and apply the distributed population dynamics derived in Section III-B. Notice that all nodes satisfy the information constraint (this fact is not possible by using the classic population dynamics). Results are shown in Figure 4 considering an initial condition $x_{i}(0)=\frac{1}{50}$, for all $i=1, \ldots, 50$. First and fourth row of Figure 4 show that $x(t)$ satisfies the problem constraints for all time, i.e., $x_{i}(t)$ remains nonnegative, for all $i=1, \ldots, 50$; and $\sum_{i=1}^{50} x_{i}(t)=1$. Furthermore, third row of Figure 4 shows that all distributed dynamics increase the objective function $V(x)$. However, only DRD, DSD, and DPD reach the optimum value (which are depicted in dashed red line). According to the second row of Figure 4, DRD, DSD, and DPD equalize the fitness functions' values in steady state, i.e., these dynamics converge to a Nash equilibrium. This behavior is consistent with the results stated in Theorems 3 and 4 since $V(x)$ corresponds to a strictly concave potential function, i.e., $F$ is a full potential and stable game. Moreover, convergence time varies from one dynamic to another. DLD shows the fastest time response while the convergence of DRD is the slowest.

The academical example proposed in Equation (8) can be adapted to model practical optimization problems. For instance, let us consider the economic dispatch of distributed generators reported in [15]. This problem is stated as follows: consider a set of $n$ generators that are connected to the electric distribution network. These generators have to supply a certain load denoted by $L$. Therefore, $\sum_{i=1}^{n} p_{i}=L$, where $p_{i} \geq 0$ is the power supplied by the $i$ th generator. The cost of production of $p_{i}$ is given by a quadratic function $c_{i}\left(p_{i}\right)=\alpha_{i}+\beta_{i} p_{i}+\gamma_{i} p_{i}^{2}$, where $\alpha_{i}, \beta_{i}, \gamma>0$ are parameters associated with the $i$-th generator. The goal of the economic dispatch problem is to minimize the total cost of energy production, which is given by $J\left(p_{1}, \ldots, p_{n}\right):=\sum_{i=1}^{n} c_{i}\left(p_{i}\right)$. Mathematically, this problem is formulated as follows:

$$
\begin{array}{ll}
\min & J\left(p_{1}, \ldots, p_{n}\right):=\sum_{i=1}^{n} \alpha_{i}+\beta_{i} p_{i}+\gamma_{i} p_{i}^{2} \\
\text { s.t. } & \sum_{i=1}^{n} p_{i}=L, \text { and } p_{i} \geq 0 \text { for all } i=1, \ldots, n .
\end{array}
$$

Notice that the formulation of problems (8) and (9) are similar. Indeed, if we let the generators be the strategies of a game, and the power supplied by the $i$ th generator be the amount of population playing the strategy $i$. Then, it is possible to model the economic dispatch of distributed generators as a population game, where the fitness functions are given by the marginal cost of energy production, i.e., $f_{i}=\frac{\partial J}{\partial p_{i}}$, for all $i=1, \ldots, n$. In this regard, we can address the economic dispatch of distributed generators by using the same procedure described in the solution of the optimization problem (8). This approach is appealing, specially in scenarios where the number of distributed generators is large.

\section{B. Classic Population Games: Bad Rock-Paper-Scissors with a Twin}

To evaluate the behavior of a non-well mixed population involved in a strategic interaction, we use a classic game called "bad rock-paper-scissor with a twin" (adapted from [3]). Moreover, we compare the performance of the distributed dynamics proposed in Section III-B with their classic counterpart (i.e., assuming a well-mixed population).

The bad rock-paper-scissor with a twin preserves the same rules as the rock-paper-scissors game. The difference is that the losing strategies have double penalty, i.e., this is not a zero-sum game. Moreover, another strategy called "twin" is added, which earns the same payoff as scissors. Summarizing, this game can be represented by the following payoff matrix

$$
A=\left(\begin{array}{rrrr}
0 & -2 & 1 & 1 \\
1 & 0 & -2 & -2 \\
-2 & 1 & 0 & 0 \\
-2 & 1 & 0 & 0
\end{array}\right),
$$

where the first, second, third, and fourth column/row corresponds to rock, paper, scissors, and twin, respectively. In this case, the fitness functions are given by $F(x)=A x$.

Evolution of the population state is shown in Figure 5 under distributed (first row) and classic (second row) dynamics. For the distributed case, we use a path graph. It can be noticed a similar behavior between the trajectories obtained by using the classic population dynamics and its distributed counterpart. 

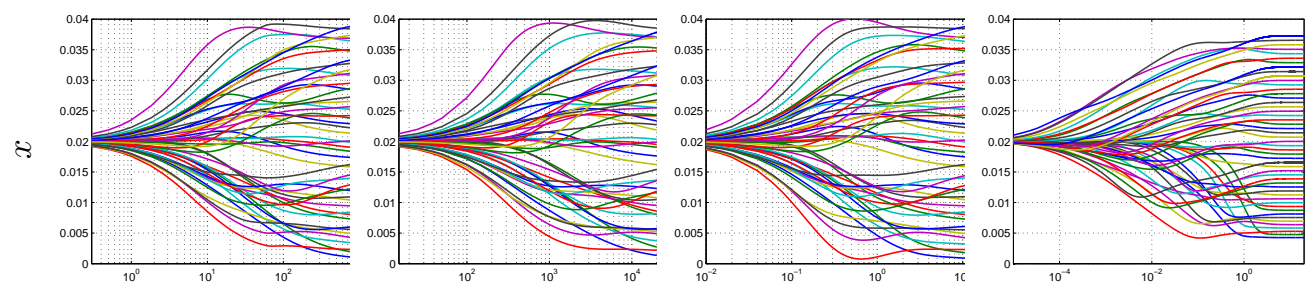

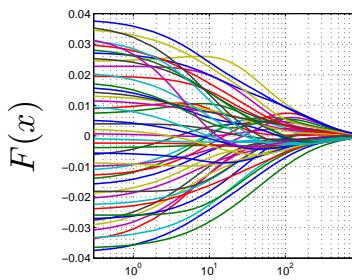

time [s]

Smith

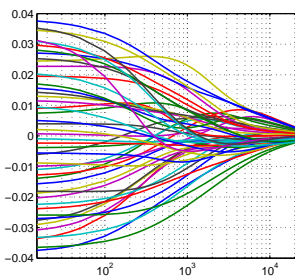

time [s]

Replicator

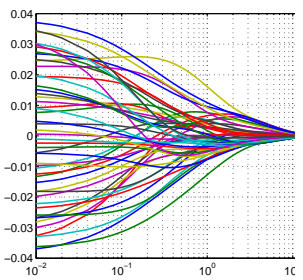

time [s]

Projection

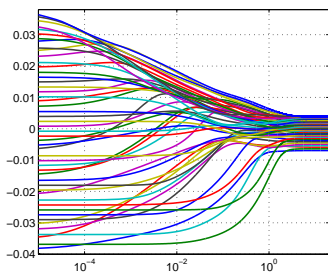

time [s]

Logit $(\eta=0.005)$

Figure 4. Evolution under different distributed population dynamics: states (1st row), fitness functions (2nd row), objective function (3rd row), equality constraint (4th row).

DSD, DRD, and DPD exhibit oscillations around the set of Nash equilibria (depicted in red). Indeed, a limit cycle emerges in each case. The main difference is that the limit cycle lies in the plane orthogonal to the set of Nash equilibria for the classic dynamics while it is not orthogonal for the distributed case. Additionally, the angle formed by the plane containing the limit cycle and the set of Nash equilibria is the same for DSD, DRD, and DPD. On the other hand, both DLD and classic logit dynamics reach the same equilibrium point. However, this rest point does not belong to the set of Nash Equilibria. We notice the fact that this characteristic has been reported before in the literature related to classic logit dynamics (e.g., see [3]).
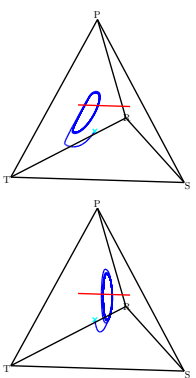

Smith
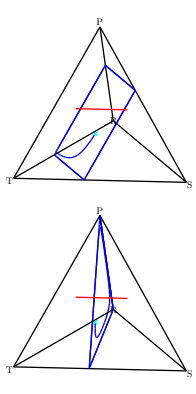

Replicator
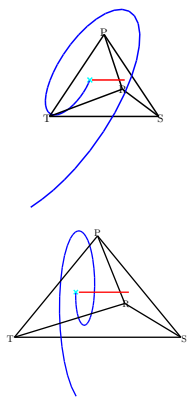

Projection
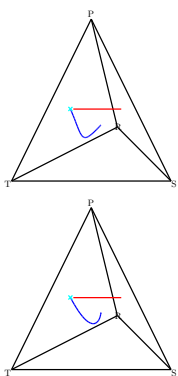

Logit $(\eta=0.8)$
Figure 5. Distributed (up) and classic (bottom) population dynamics applied to bad $\operatorname{rock}(\mathrm{R})-\operatorname{paper}(\mathrm{P})-$-scissors(S) with a twin(T).

\section{Distributed Control of Dynamical Systems}

Finally, we design a distributed controller for the optimal transportation of drinking water ${ }^{1}$ (relationship between distributed control and games has been pointed out in several papers, e.g., [31], [32]). The system is composed by $n$ coupled tanks as shown in Figure 6. The arrows in the graphical

\footnotetext{
${ }^{1}$ Parameters of this academical problem have been scaled from real transportation of drinking water problems.
}

representation show how flow directions are. Each tank has an outflow given by an unknown demand considered as a disturbance that is denoted by $d_{i}$, and an inflow $u_{i}$ from a limited water source, i.e., the control outputs are subject to a constraint given by $\sum_{i=1}^{n} u_{i} \leq K$, where $K$ is the total available resource. The mentioned inflows are controlled by $x_{i}$ that determines a percentage of the total resource $K$, then $u_{i}=K x_{i}$, and $\sum_{i=1}^{n} x_{i}=1$. We assume that there are local controllers at the valves to guarantee the desired inflow according to the signal $x_{i}$. The dynamics for this system are

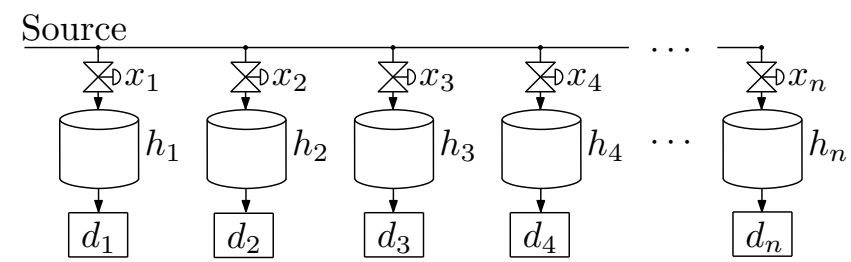

Figure 6. Simple drinking water system with a unique resource and unknown demands.

as follows,

$$
\begin{aligned}
& \frac{d h_{1}}{d t}=u_{1}-\sqrt{\rho g h_{1}}-d_{1} \\
& \frac{d h_{i}}{d t}=u_{i}+\sqrt{\rho g h_{i-1}}-\sqrt{\rho g h_{i}}-d_{i}, i=2, \ldots, n-1 \\
& \frac{d h_{n}}{d t}=u_{n}+\sqrt{\rho g h_{n-1}}-d_{n},
\end{aligned}
$$

where $h_{i}$ is the water level of the $i$ th tank, $\rho$ is the density of the fluid, and $g$ is the gravity. The proposed example considers the case of 4 tanks, and the control objective is to maintain the water level of each tank at a safety value of reference, which is given by the company in charge of the management of the network. This safety value is obtained according to the demand that each tank supplies. For this particular example the safety reference is established at $0.5 \mathrm{~m}$, and the unknown demand 
profile at each node during two days is shown in Figure 7 (adapted from [33]).

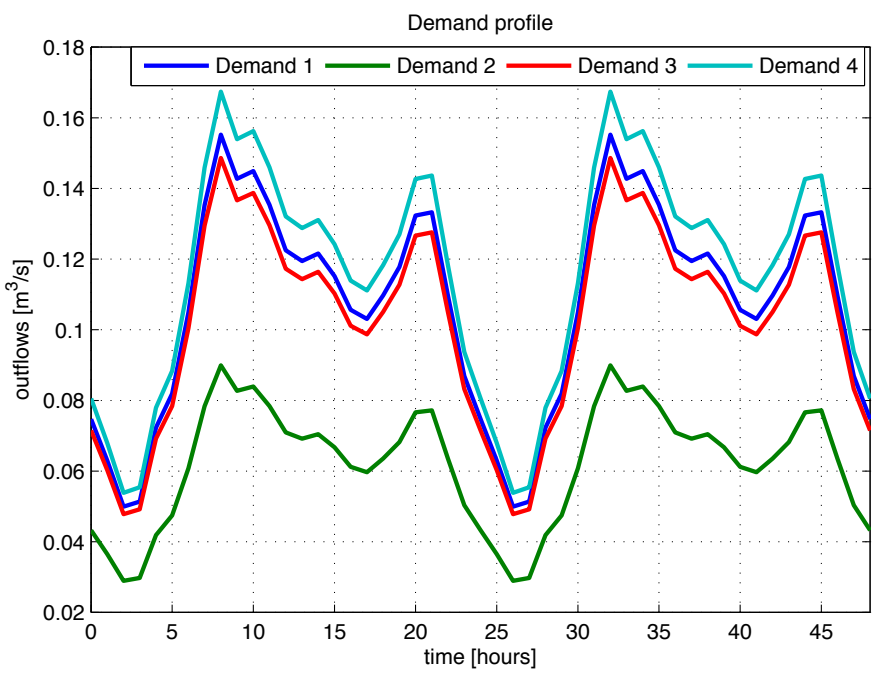

Figure 7. Demand profile $\left(d_{1}, d_{2}, d_{3}\right.$ and $\left.d_{4}\right)$ during 2 days for the 4 tanks case.

To control this plant using population dynamics, we propose the following analogy: the population is related to the available water, the strategies correspond to the tanks. Therefore, the population state $x$ represents the distribution of the water (in percentage) among the tanks. Additionally, the fitness functions are selected to be the error at each tank, i.e., $f_{i}=0.5-h_{i}$, for all $i=1, \ldots, n$. Notice that this fitness is appropriate since: $i$ ) more proportion of inflow is assigned to those tanks with larger error, and ii) it is known that $h_{i}$ increases as the inflow controlled by $x_{i}$ increases, i.e., the fitness function $f_{i}$ is decreasing with respect to $x_{i}$ satisfying the condition for a stable game [3]. Furthermore, consider the case in which the quantity of water required to meet the safety levels is less than the available resource. In this situation, if all the available resource is allocated in the tanks, the safety levels would be exceeded. Therefore, it is necessary to introduce an additional strategy whose corresponding population $x_{n+1}$ is used as a slack variable. The fitness function for this slack variable is $f_{n+1}=0$. Hence, if the level in the $i$ th tank is higher than the safety level (i.e., $f_{i}<0$ ), it is more profitable to allocate resource to the slack variable than to the $i$ th tank.

Figure 8 shows the control performance considering full information in the classic Smith dynamics, i.e., that at each point of the network, the information related to all the system is available to make decisions. Figure 9 shows the performance of a distributed controller designed based on DSD. The information graph considered for this example is a path graph, i.e., that the $i$ th tank only has information about the $(i-1)$ th and the $(i+1)$ th tanks. First, notice that in this particular problem with four control actions and one slack variable, the classical Smith dynamics with full information require 10 communication links. In contrast, the distributed population dynamics approach for a problem with four control actions, one slack variable, and a path configuration just require four communication links.
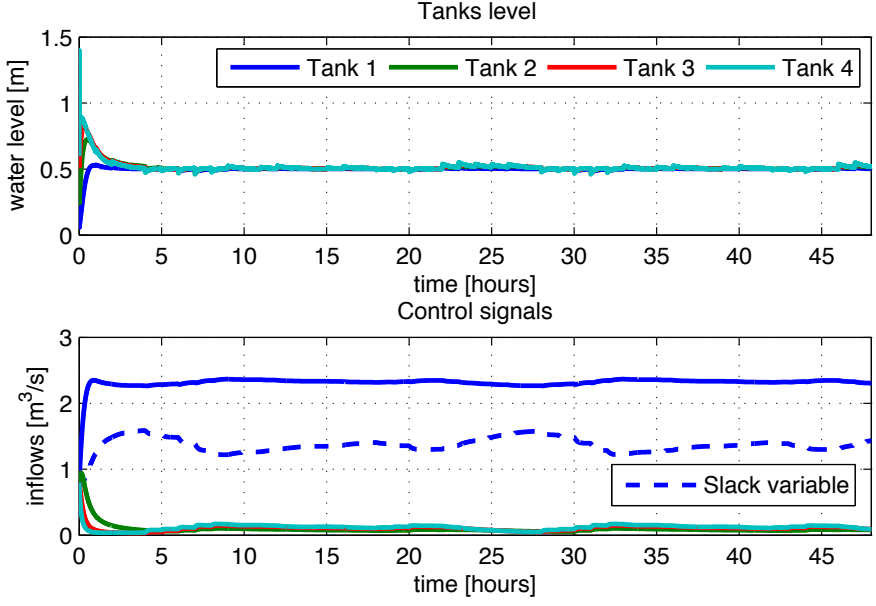

Figure 8. System states evolution for a controller with full information.
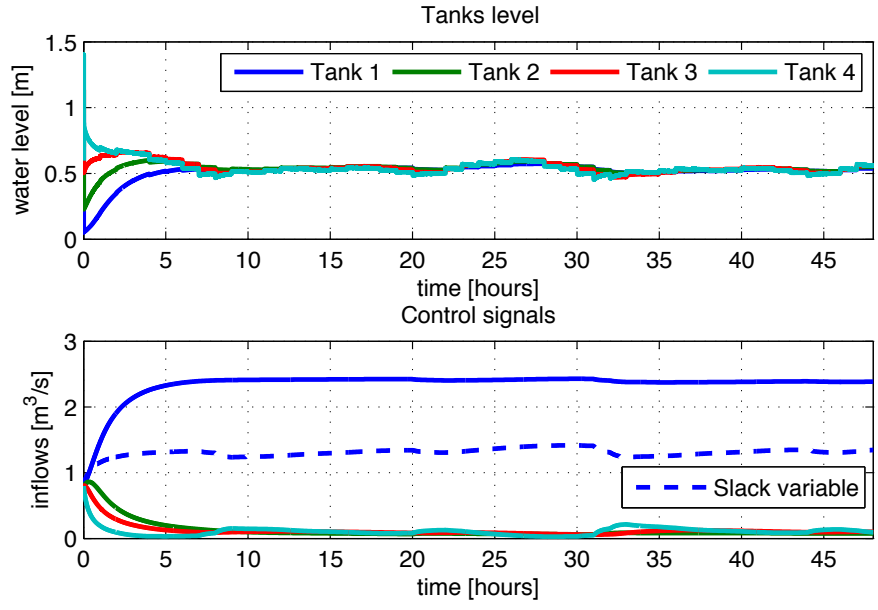

Figure 9. System states evolution for a distributed population dynamics based controller.

Regarding control performance, results show that the control objective is achieved with both the Smith dynamics controller with full information and with the DSD controller by using partial information despite the hard and unknown outflow disturbances at each tank. Furthermore, it can be noticed that the settling time of the distributed controller is not far from the one obtained with full information. Additionally, once the system achieves the set-point, both controllers have similar behavior showing the well performance of the distributed population dynamics approach.

\section{DISCUSSION}

This paper proposes a methodology to generate distributed population dynamics from revision protocols considering different population structures. The core of the proposed method lies in a generalization of the mean dynamics for non-wellmixed populations. This novel concept provides us a tool to deal with information constraints related to the strategic interactions of the individuals that conform the population. Allowed agents' interactions within the population are described by means of an undirected graph. Following this convention, we have shown that well-mixed populations are characterized 
by complete graphs, while non-well-mixed populations are represented by non-complete graphs. The main implication of this feature is the possibility to derive population dynamics that only use local information to evolve, i.e., distributed population dynamics.

The appealing features of distributed population dynamics have been exploited in some application examples that illustrate their usefulness. We have shown that distributed population dynamics can be used in the design of constrained optimization algorithms, and the synthesis of control systems for problems requiring non-centralized schemes. Compared to other distributed techniques such as dual decomposition methods [34], the implementation of distributed population dynamics does not need the inclusion of a centralized coordinator. This property is important because it reduces the cost of the required communication infrastructure. Furthermore, some families of distributed population dynamics (e.g., DRD, DSD, and DLD) naturally address non-negativity constraints (see Theorem 2) that emerges in a variety of problems due to physical limitations. For instance, in scheduling problems, the time allocated to each task cannot be negative. Although non-negativity constraints are tackled in other distributed approaches by means of barrier functions, it is known that the use of barrier functions carries out some problems related to the convergence rate and the accuracy of the solution, especially for large-scale problems [35].

Different from population games, normal form games are also employed in the literature to address engineering applications. Even though both frameworks are similar, they have some important differences. For instance, in population games, the players are characterized by a continuum of mass, while in normal form games, each player is individually treated. This difference makes population dynamics suitable to be applied in problems that involve a large number of players that have the same characteristics; for instance, in multiple access control for communication systems [9]. On the other hand, It is worth noting that most research effort has been devoted to study distributed learning techniques in normal form games [36], [23]. However, distributed methods for addressing population games have been somewhat unexplored. In this regard, the results shown in this paper aim to motivate the use of population dynamics in applications that require non-centralized schemes. An important advantage of distributed population dynamics compared to distributed learning algorithms for normal form games has been pointed out in [37], where the author suggests that classic learning techniques fail in applications that include a coupled constraint that involves all the decision variables. Under this scenario, distributed population dynamics become a proper alternative since their trajectories evolve to the optimal solution satisfying the coupled constraint associated to the simplex $\Delta$ as shown in Theorems $2-4$.

This property can be exploited in resource allocation problems among a set of entities, which are given by the following formulation:

$$
\begin{aligned}
\max & V(x) \\
\text { s.t. } & \sum_{i=1}^{n} x_{i}=X \\
& x_{i} \geq 0, \text { for all } i=1, \ldots, n,
\end{aligned}
$$

where $x_{i}$ is the resource allocated to the $i$ th entity, $X$ is the available resource, and $V(x)$ is a concave utility function. Notice that, distributed population dynamics can be used to solve this problem in a distributed way (cf. Theorem 3). Indeed, an advantage of population-dynamics-based techniques is that they provide feasible solutions at any time of the optimization process. This property makes distributed population dynamics appropriate to be applied in online control schemes as in the example of the optimal transportation of drinking water (c.f. Section V-C). Moreover, from the proof of Theorem 3, we can conclude that, in optimization problems described by potential games (as is the case of the resource allocation problem shown in Equation (10)), the associated utility function is permanently increasing under distributed population dynamics. Therefore, if the optimization process needs to be stopped before obtaining the optimal solution, the founded solution is at least suboptimal.

Simulations have illustrated the equivalence between the steady state behavior of the proposed distributed dynamics and classic population dynamics. Moreover, regarding the transient performance, simulations have also shown that there exists a relationship between the convergence rate and the algebraic connectivity of the underlying communication graph. The larger the algebraic connectivity is, the faster the transient response of the corresponding dynamics. Indeed, the fastest response is obtained by using a complete-graph, i.e., by using classic population dynamics. Similarly to other distributed schemes, the dependence of the performance on the graph connectivity evidences a tradeoff between the number of communication links and how fast the optimal solution is reached.

\section{CONCLUSIONS}

We have generalized a methodology to generate distributed population dynamics from the distributed mean dynamics and different revision protocols. To illustrate the method, we have derived four distributed population dynamics, i.e., the distributed Smith dynamics, the distributed replicator dynamics, the distributed projection dynamics and the distributed logit dynamics. We have proved that the distributed population dynamics presented in this work exhibit mass conservation and convergence to Nash equilibria in the same way as classic population dynamics do. Finally, we have presented some applications of the proposed dynamics in the design of distributed optimization algorithms, solution of classic games, and the synthesis of controllers for multi-variable system. Simulation results have shown that distributed population dynamics exhibit an appropriate performance and provide optimal solutions despite the lack of full information. Thus, distributed population dynamics are promising to address problems requiring non-centralized information structures. This fact is relevant since distributed problems are becoming more common in many fields, especially when large-scale and complex systems are involved.

\section{ACKNOWLEDGEMENTS}

We would like to thank Professor Kevin M. Passino for his valuable inputs. 


\section{REFERENCES}

[1] J. W. Weibull, Evolutionary Game Theory. London, England: The MIT Press, 1997.

[2] J. Hofbauer and K. Sigmund, Evolutionary games and population dynamics. Cambridge University Press, Cambridge, 1998.

[3] W. H. Sandholm, Population games and evolutionary dynamics. Cambridge, Mass. MIT Press, 2010.

[4] M. J. Fox and J. S. Shamma, "Population games, stable games, and passivity.," in Proceedings of the IEEE 51st Conference on Decision and Control (CDC), pp. 7445-7450, 2012.

[5] J. Marden and J. S. Shamma, "Game theory and distributed control," in Handbook of Game Theory Vol. 4 (H. Young and S. Zamir, eds.), pp. 861-900, North Holland, 2014

[6] S. Mannor and J. S. Shamma, "Multi-agent learning for engineers," Artificial Intelligence, special issue on Foundations of Multi-Agent Learning, vol. 171, pp. 417-422, May 2007.

[7] N. Li and J. Marden, "Designing games for distributed optimization with a time varying communication graph," in Proceedings of the IEEE 51st Conference on Decision and Control (CDC), pp. 7764 - 7769, 2012.

[8] J. Barreiro-Gomez, N. Quijano, and C. Ocampo-Martinez, "Constrained distributed optimization based on population dynamics," in Proceedings of the 53rd IEEE Conference on Decision and Control (CDC), pp. 42604265,2014

[9] H. Tembine, E. Altman, R. El-Azouzi, and Y. Hayel, "Evolutionary games in wireless networks," IEEE Transactions on Systems, Man, and Cybernetics, Part B: Cybernetics, vol. 40, no. 3, pp. 634-646, 2010.

[10] G. Obando, A. Pantoja, and N. Quijano, "Building Temperature Control based on Population Dynamics," IEEE Transactions on Control Systems Technology, vol. 22, no. 1, pp. 404-412, 2014

[11] S. Berninghaus and H. Haller, "Local interaction on random graphs," Games, vol. 1, no. 3, pp. 262-285, 2010.

[12] C. Alós-Ferrer and S. Weidenholzer, "Imitation, local interactions, and efficiency," Economics Letters, vol. 93, pp. 163-168, 2006.

[13] B. Gharesifard and J. Cortes, "Distributed convergence to Nash equilibria by adversarial networks with directed topologies," in Proceedings of the American Control Conference, pp. 5881-5886, 2012.

[14] O. Boussaton and J. Cohen, "On the distributed learning of Nash equilibria with minimal information," in Proceedings of the 6th International Conference on Network Games, Control, and Optimization, pp. 30-37, 2012.

[15] A. Pantoja and N. Quijano., "Distributed optimization using population dynamics with a local replicator equation.," in Proceedings of the 51st IEEE Conference on Decision and Control (CDC), pp. 3790-3795, 2012.

[16] J. Hofbauer and W. H. Sandholm, "Stable games and their dynamics," Journal of Economic Theory, vol. 144, no. 4, pp. 1665-1693, 2009.

[17] L. Moreau, "Stability of multiagent systems with time-dependent communication links," IEEE Transactions on Automatic Control, vol. 50, no. 2, pp. 169-182, 2005.

[18] R. Olfati-Saber, J. A. Fax, and R. M. Murray, "Consensus and cooperation in networked multi-agent systems," Proceedings of the IEEE, vol. 95, no. 1, pp. 215-233, 2007.

[19] W. Ren and R. Beard, "Consensus seeking in multiagent systems under dynamically changing interaction topologies," IEEE Transactions on Automatic Control, vol. 50, no. 5, pp. 655-661, 2005.

[20] N. Li and J. R. Marden, "Designing games for distributed optimization," IEEE Journal of Selected Topics in Signal Processing, vol. 7, no. 2, pp. 230-242, 2013. special issue on adaptation and learning over complex networks.

[21] J. R. Marden, S. D. Ruben, and L. Y. Pao, "A Model-Free Approach to Wind Farm Control Using Game Theoretic Methods," IEEE Transactions on Control Systems Technology, vol. 21, no. 4, pp. 1207-1214, 2013.

[22] D. Fudenberg and J. Tirole, Game Theory. Cambridge, MA: MIT Press, 1991.

[23] G. Arslan and J. Shamma, "Distributed convergence to Nash equilibria with local utility measurements," in Proceedings of the Decision and Control, 2004. CDC. 43rd IEEE Conference on, vol. 2, pp. 1538-1543, 2004.

[24] I. Bomze, M. Pelillo, and V. Stix, "Approximating the maximum weight clique using replicator dynamics," IEEE Transactions on Neural Networks, vol. 11, no. 6, pp. 1228-1241, 2000.

[25] J. Poveda and N. Quijano, "Dynamic bandwidth allocation in wireless networks using a shahshahani gradient based extremum seeking control," in proceedings of the 2012 6th International Conference on Network Games, Control and Optimization (NetGCooP), pp. 44-50, IEEE, 2012.
[26] E. Mojica-Nava, C. A. Macana, and N. Quijano, "Dynamic population games for optimal dispatch on hierarchical microgrid control," Systems, Man, and Cybernetics: Systems, IEEE Transactions on, vol. 44, no. 3, pp. 306-317, 2014.

[27] A. Pantoja and N. Quijano, "A population dynamics approach for the dispatch of distributed generators," IEEE Transactions on Industrial Electronics, vol. 58, no. 10, pp. 4559-4567, 2011.

[28] J. I. Poveda and N. Quijano, "Shahshahani gradient-like extremum seeking," Automatica, vol. 58, pp. 51-59, 2015.

[29] J. Barreiro-Gomez, N. Quijano, and C. Ocampo-Martinez, "Distributed control of drinking water networks using population dynamics: Barcelona case study," in Proceedings of the 53rd IEEE Conference on Decision and Control (CDC), pp. 3216-3221, 2014.

[30] S. Bornholdt and H. G. Schuster, Handbook of graphs and networks, vol. 2. Wiley Online Library, 2003.

[31] J. R. Marden, G. Arslan, and J. S. Shamma, "Cooperative control and potential games," IEEE Transactions on Systems, Man and Cybernetics. Part B: Cybernetics, vol. 39, pp. 1393-1407, December 2009.

[32] J. Wang and N. Elia, "A control perspective for centralized and distributed convex optimization.," in Proceedings of the 50th IEEE Conference on Decision and Control and European Control Conference (CDC-ECC), pp. 3800-3805, 2011.

[33] C. Ocampo-Martinez, D. Barcelli, V. Puig, and A. Bemporad, "Hierarchical and decentralised model predictive control of drinking water networks: application to Barcelona case study," IET Control Theory and Applications, vol. 6, p. 62, January 2012.

[34] D. Palomar and M. Chiang, "A tutorial on decomposition methods for network utility maximization," IEEE Journal on Selected Areas in Communications, vol. 24, no. 8, pp. 1439-1451, 2006.

[35] P. A. Jensen and J. F. Bard, Operations research models and methods. John Wiley \& Sons Incorporated, 2003.

[36] J. R. Marden, G. Arslan, and J. S. Shamma, "Joint strategy fictitious play with inertia for potential games," IEEE Transactions on Automatic Control, vol. 54, no. 2, pp. 208-220, 2009.

[37] J. R. Marden, "State based potential games," Automatica, vol. 48, no. 12, pp. 3075-3088, 2012. 